\title{
Tipo de cambio real en Bolivia: equilibrio y desalineamientos
}

\section{Real Exchange Rate in Bolivia: Equilibrium and misalignment}

Sergio Cerezo Aguirre* Denise Salazar Gómez**

\section{Resumen $^{1}$}

Conocer el comportamiento del tipo de cambio real respecto a su equilibrio (interno y externo) permite determinar la existencia de des-alineamientos, y a partir de ello plantear recomendaciones sobre el diseño de políticas. En esa línea, el presente documento aplica cuatro métodos distintos para estimar el TCR de equilibrio en Bolivia, en algunos casos para un horizonte temporal y en otros para un periodo en particular. Los métodos revisados fueron: a) Filtro Estadístico - Hodrick y Prescott, b) Modelo de Comportamiento del TCR, c) Método del Equilibrio Macroeconómico y d) Método de Sostenibilidad Externa. Los resultados de todos estos métodos coinciden y sugieren una subvaluación cambiaria promedio de $0.69 \%$ para 2011, a pesar de que cada metodología considera distintas simplificaciones conceptuales e información diversa.

Palabras clave: Tipo de cambio real de equilibrio, Bolivia

\footnotetext{
* Docente de la Carrera de Economía de la Universidad Católica Boliviana y miembro de la SEBOL. Contacto: scerezoa@gmail.com

** Miembro de la SEBOL .Contacto: denise_sg@yahoo.com
}

1 Las opiniones vertidas en este documento son de responsabilidad exclusiva de los autores 


\begin{abstract}
The behavior of the real exchange rate with respect to its equilibrium (internal and external) follow determines the existence of desalignment, and from these developing recommendations for policy design. In this case, this paper makes a methodological review of four different methods to estimate the equilibrium real exchange rate and applies then to Bolivia, in some cases, for a time horizon and others for a particular period. The methods reviewed were: a) Hodricky Prescott filter, b) Estimation of a Behavioral Equilibrium Exchange Rate, c) Macroeconomic Balance Approach and d) External Sustainability Approach. The results of the methods agree and suggest an undervalued exchange rate (average) of 0.69\% for 2011, even though each methodology considers different conceptual simplifications and information.
\end{abstract}

Keywords: Equilibrium real exchange rates, Bolivia

\title{
Clasificación/ Classification JEL: F3, F31
}

\section{Introducción}

Conocer el tipo de cambio de equilibrio es importante para el diseño e implementación de la política económica. Su relevancia se justifica tanto por su importancia en la asignación de los recursos entre sectores comerciables y no comerciables como en la definición de estrategias de inversión y endeudamiento externo. Asimismo, conocer la existencia de des-alineamientos del tipo de cambio real, es decir, determinar y cuantificar la diferencia entre el tipo de cambio observado y aquél que sugiere sus fundamentos (Tipo de Cambio Real de Equilibrio, TCRE), permite plantear recomendaciones de política para ajustar este precio relativo.

De una revisión bibliográfica se puede resaltar que existen varios métodos para calcular el TCRE. Sobre el tema, Isard (2007) sostiene que cada método implica simplificaciones conceptuales y/o estimaciones que no siempre son precisas; $y$, a veces, un método distinto genera estimaciones cuantitativas muy distintas de los TCRE. Por esta razón, no se puede confiar plenamente en las estimaciones formuladas mediante la aplicación de un solo método. En este sentido, es altamente deseable contar con distintos métodos para estimar posibles desalineamientos del TCR. 
La importancia en la estimación del TCRE ha generado diversas metodologías. Por ejemplo, el Fondo Monetario Internacional (FMI), en base al trabajo realizado por el Grupo Consultivo sobre Tipo de Cambio (CGER, Consultative Group on Exchange Rate Issues), establecida a mediados de los años 1990 y cuya última revisión metodológica se realizó en 2006, evalúa el comportamiento del tipo de cambio en economías avanzadas y emergentes. Bajo este entorno, en Lee et al. (2006) y Lee et al. (2008) se distinguen tres metodologías para la supervisión del tipo de cambio: el equilibrio macroeconómico $(M B$, macroeconomic balance), el tipo de cambio real de equilibrio (REER, equilibrium real Exchange rate) y la sostenibilidad externa (ES, external sustainability).

El presente documento, en base a la aplicación de las tres metodologías descritas anteriormente, procurará identificar posibles des-alineamientos cambiarios (subvaluaciones o sobrevaluaciones ${ }^{2}$ ); y evaluar empíricamente qué fundamentos lo determinan en el largo plazo. En síntesis, los métodos aplicados en el presente documento son: a) Modelo de Comportamiento del Tipo de Cambio Real (BEER, por sus siglas en inglés), b) Método del Equilibrio Macroeconómico, y c) Método de Sostenibilidad Externa. En la sección 2 se evaluará cada método para el caso de Bolivia y en la sección 3 se presentarán las conclusiones.

\section{Enfoques para estimar el tipo de cambio real de equilibrio}

\subsection{Filtro estadístico Hodrick y Prescott (1980)}

El filtro estadístico de Hodrick y Prescott (1980) es un filtro usualmente empleado para estimar el componente tendencial (largo plazo) de las series ${ }^{3}$. La aplicación de este filtro al tipo de cambio real de equilibrio da cuenta de tres periodos de des-alineamiento cambiario. Estos periodos son 1994-1995, con una subvaluación, 2002 y 2008-2009 con una sobrevaluación cambiaria (Gráfico 1).

2 De acuerdo con la definición de TCR empleada en este trabajo, se define una subvaluación como momentos en los cuales el TCR se halla por encima de su nivel de equilibrio; mientras que una sobrevaluación responde a aquellos momentos en los que el TCR se halla por debajo de su nivel de equilibrio.

3 El landa empleado para este ejercicio es uno calibrado para la economía boliviana, landa = 7185 . 


\section{Gráfico 1: Descompocisión del tipo de cambio real Filtro de Hodrick y Prescott}

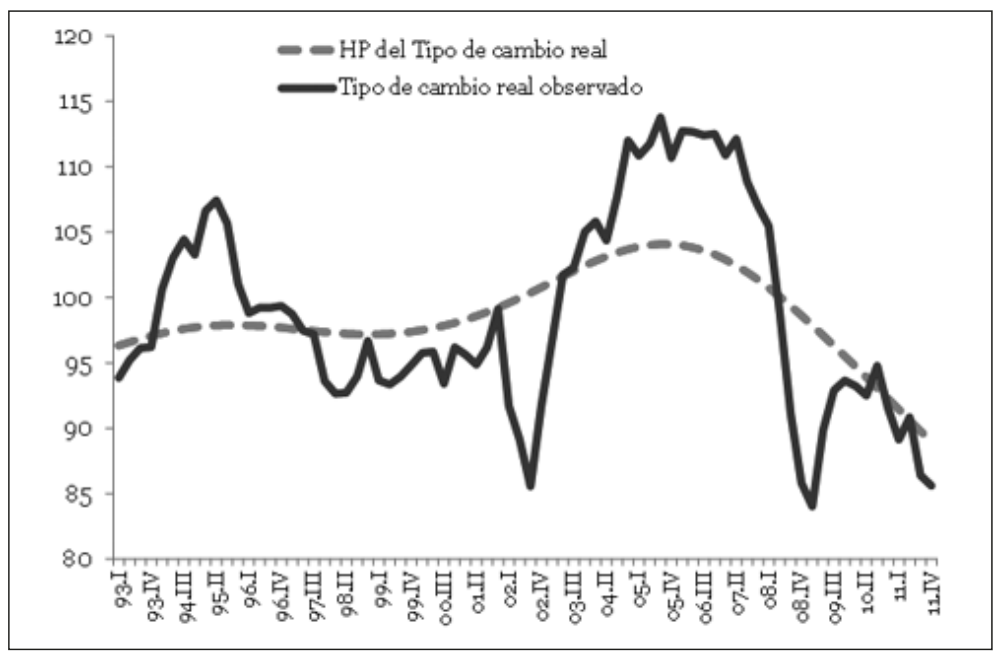

Fuente: Elaboración propia

Como se verá más adelante, estos periodos de des-alineamiento coinciden con los encontrados bajo el Método de Comportamiento del TCR. Específicamente para 2011, este método estima una subvaluación cambiaria mínima de -0.78\%. Sin embargo, la literatura económica ha cuestionado bastante este método para extraer el componente de largo plazo de una serie, además de carecer de sustento económico, por lo que en las siguientes secciones exploraremos otras visiones que contienen componentes analíticos y macroeconómicos.

\subsection{Modelo de Comportamiento del Tipo de Cambio Real}

El Modelo de Comportamiento del Tipo de Cambio Real (Behavioral Equilibrium Exchange Rate, BEER) es uno de los métodos comúnmente empleados en la estimación del TCRE, el cual se basa en modelos econométricos para establecer los fundamentos que explican el tipo de cambio real, para luego poder estimar el grado de des-alineamiento cambiario. La evidencia internacional que aplica esta metodología puede encontrarse en Ades et. al. (1999), Hoffman y MacDonald (2003), MacDonald y Ricci (2003), Choudhri y Khan (2004), Miyajima (2005), entre otros. 
En Bolivia existen documentos de investigación que, bajo esta metodología, consideran como fundamentos del tipo de cambio real a los términos de intercambio, la apertura comercial, los flujos de capital, el balance fiscal, la productividad y la política comercial. El Cuadro 1 provee un resumen de los estudios más recientes, en el cual se presenta simplemente el efecto (+) o (-) de la variable fundamento sobre el tipo de cambio real.

\section{Cuadro 1}

Estudios sobre los determinantes del tipo de cambio real en Bolivia

\begin{tabular}{lcccccc}
\hline \hline \multicolumn{7}{c}{ Loray } \\
Autores & Aguilar & Mendieta & $\begin{array}{c}\text { Cerruti y } \\
\text { Mansilla }\end{array}$ & $\begin{array}{c}\text { Bello, } \\
\text { Heresi y } \\
\text { Pineda }\end{array}$ & $\begin{array}{c}\text { Cerezo, } \\
\text { Humerezy } \\
\text { Cossio }\end{array}$ \\
\hline Año & 2000 & 2003 & 2007 & 2008 & 2010 & 2010 \\
\hline \multicolumn{7}{c}{ Fundamentos } \\
\hline Balance fiscal & \multicolumn{7}{c}{ Estudios } \\
Apertura comercial & $(+)$ & $(+)$ & $(+)$ & $(+)$ & $(+)$ & $(+)$ \\
Flujos de capital & no & $(+)$ & no & no & $(+)$ & $(+)$ \\
Términos de intercambio & $(-)$ & $(-)$ & $(-)$ & $(-)$ & no & no \\
Política comercial & $(-)$ & $(-)$ & no & $(-)$ & $(-)$ & $(-)$ \\
Productividad & $(-)$ & $(-)$ & no & no & no & no \\
\hline \hline
\end{tabular}

Este documento estima, en base a cuatro modelos econométricos de cointegración, el tipo de cambio real de equilibrio en Bolivia combinando el siguiente grupo de variables: tipo de cambio real multilateral, precio de exportación del gas natural, reservas internacionales netas (RIN), términos de intercambio, balance fiscal y balanza comercial, estos dos últimos como porcentaje del $\mathrm{PIB}^{4}$. A diferencia de otras investigaciones aplicadas en Bolivia, el presente documento incluye y prueba la significancia de las RIN, cuya evolución refleja las presiones que pueden existir en el mercado cambiario dado el régimen cambiario vigente (crawling peg). Por otra parte, se incluye el precio de exportación del gas natural (que es el precio promedio ponderado de exportación a Argentina y Brasil), debido a que en los últimos años la evolución de las cuentas externas y fiscales han sido influidas por los recursos de este sector (Cuadro 2).

4 En el Anexo 1 se presenta un análisis de estacionariedad de cada variable. En ésta se muestra que todas presentan raíz unitaria, por lo que es posible buscar una relación de cointegración. Por su parte, el Anexo 2 muestra los resultados de las pruebas de cointegración de Johansen, que proporcionan evidencia de que existe un único vector de cointegración entre las diferentes combinaciones de las variables. 
Cuadro 2

Determinantes del tipo de cambio real: estimación del vector de cointegración

\begin{tabular}{|c|c|c|c|c|}
\hline Descripción & (1) & (2) & (3) & (4) \\
\hline Primera observación & $1999 \mathrm{~T}_{4}$ & $1999 \mathrm{~T}_{4}$ & $1990 \mathrm{~T}_{1}$ & $1990 \mathrm{~T}_{1}$ \\
\hline Últimaobservación & $2011 \mathrm{~T}_{4}$ & $2011 \mathrm{~T}_{4}$ & $2011 \mathrm{~T}_{4}$ & $2011 \mathrm{~T}_{4}$ \\
\hline Número de observaciones & 49 & 49 & 84 & 84 \\
\hline Ln(Balance Fiscal/PIB) & 1.59 & 1.74 & 205 & 4.04 \\
\hline Error Estándar & $(0.29)$ & $(0.36)$ & $(0.66)$ & $(0.75)$ \\
\hline Ln(Balanza Comercial/PIB) & -1.56 & -0.94 & -0.92 & -1.74 \\
\hline Error Estándar & $(0.23)$ & $(0.08)$ & $(0.37)$ & $(0.44)$ \\
\hline Ln(Términos de Intercambio) & & & -0.63 & -0.46 \\
\hline Error Estándar & & & $(0.19)$ & $(0.19)$ \\
\hline Ln(Precio Exportación Gas) & -0.28 & -0.70 & & \\
\hline Error Estándar & $(0.10)$ & $(0.12)$ & & \\
\hline $\operatorname{Ln}(\mathrm{RIN})$ & & -0.40 & & -0.16 \\
\hline Error Estándar & & $(0.12)$ & & $(0.08)$ \\
\hline Test LM Correlación serial & 0.20 & 0.17 & 0.25 & 0.36 \\
\hline Test Jarque-Bera de normalidad & 0.02 & 0.02 & 0.10 & 0.02 \\
\hline Test de Heterocedasticidad & 0.92 & 0.51 & 0.58 & 0.64 \\
\hline Criterio de Akaike & -11.95 & $-13,06$ & -13.23 & -14.97 \\
\hline Log Verosimilitud & 385 & 426 & 674 & 762 \\
\hline
\end{tabular}

Fuente: Actualización a Cerezo y Salazar (2011)

Nota: La variable dependiente es el logaritmo del tipo de cambio real y efectivo. El tratamiento de estacionalidad para cada variable se la realizó con el X12-Arima. Todas las regresiones incluyen dos rezagos y una constante. La información trimestral para el periodo 1990-2011. Sin embargo, el modelo que incluye el precio de exportación del gas considera información trimestral para el periodo 1999-2011, debido a la disponibilidad de este precio.

De una evaluación técnica a los modelos de cointegración estimados, se encuentra que la especificación (4) explica de mejor manera el comportamiento del tipo de cambio real multilateral, el cual incluye los términos de intercambio, reservas internacionales netas, balance fiscal y balance comercial ${ }^{5}$. Los resultados reflejan que: a) una mejora en el balance fiscal ocasiona una depreciación del tipo de cambio real; en particular, ante un aumento en

5 Todas las estimaciones de los coeficientes de esta ecuación son estadísticamente significativas y tienen los signos esperados (Anexo A2) 
$1 \%$ en el balance fiscal como proporción del PIB el tipo de cambio real se deprecia en 4.04\% b) la ganancia en los términos de intercambio y el incremento en las RIN ocasionan una apreciación real, debido a que presionan a la baja el precio de la divisa y porque significan un mayor gasto agregado que presiona al alza los precios de bienes no transables. El efecto es mayor en el caso de los términos de intercambio que presentan una elasticidad de largo plazo de 0.46 , mientras que las RIN registran una elasticidad de 0.16 . Finamente, una mejora en la balanza comercial presiona a una caída en el tipo de cambio real con una elasticidad de 1.74.

La comparación del tipo de cambio real de equilibrio estimado con el observado no parece mostrar evidencia de des-alineamientos persistentes del tipo de cambio real respecto a sus fundamentos, especialmente en los últimos trimestres ${ }^{7}$. Cuantitativamente, se emplea como una medida de des-alineamiento la diferencia entre el tipo de cambio real efectivo y la estimación de su "equilibrio" de largo plazo (modelo 4). Los resultados sugieren tres periodos de des-alineamiento: subvaluación real en el periodo 1994-1995 y sobrevaluación en los periodos 2002 y 2008-2009 (Gráfico 2). Estos breves desequilibrios han estado asociados principalmente con turbulencias macroeconómicas internacionales que afectaron a algunos países que son socios comerciales y recientemente con la crisis económica internacional, que se inició aproximadamente en septiembre de 2008. De acuerdo a la estimación del tipo de cambio real de equilibrio, en los últimos años la apreciación real ha sido consistente con la evolución de sus fundamentos, y para el año 2011 se evidenció por este método una subvaluación cambiaria real de $-0.65 \%^{8}$.

6 Sin embargo, sería ilustrativo analizar la fuente de la mejora en las cuentas fiscales y verificar si está relacionada con una menor presión sobre el gasto agregado.

7 Para este ejercicio se consideraron los valores tendenciales (utilizando HP) de los determinantes en línea con todos los trabajos empíricos.

8 El intervalo de equilibrio se lo obtuvo a partir de un ejercicio de Bootstrap; sin embargo, para fines de comparación con los otros métodos vistos más adelante, en este documento se considera la estimación puntual. 


\section{Gráfico 2: Estimación del des-alineamiento cambiario para el periodo 1993-2011}

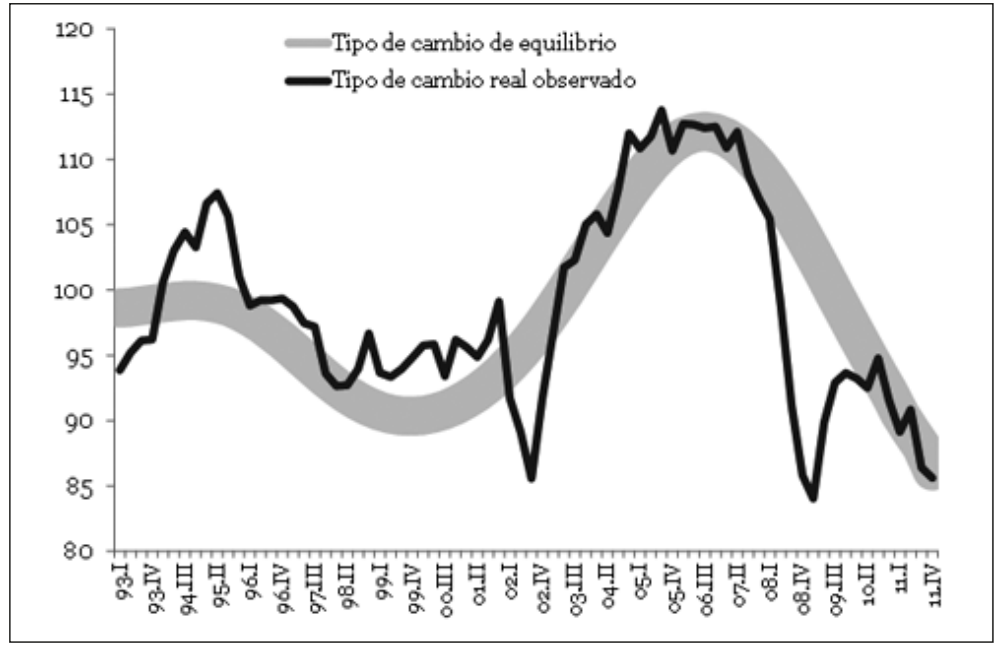

Fuente: Estimaciones propias
Nota: La banda es estimada a partir de un Bootstrap

\subsection{Método de equilibrio macroeconómico (MEM)}

Este tipo de modelos corresponde a los que Williamson (1983) denomina Fundamental Equilibrium Exchange Rate o FEER y que el FMI (2008) ha desarrollado para evaluar los tipos de cambios reales de diferentes economías. Este método consiste en encontrar un tipo de cambio real de equilibrio que sea coherente en forma simultánea con los equilibrios externo e interno de la economía. El equilibrio interno es alcanzado cuando el producto efectivo es igual al producto potencial (la brecha de producto es cero). Por otro lado, el equilibrio externo se logra cuando la cuenta corriente está en un nivel sostenible.

Según FMI (2008), el MEM tiene tres componentes básicos: a) una identidad entre el saldo en cuenta corriente (CUR) y la salida neta de capital internacional (CAP), o entre el CUR y el exceso de ahorro interno (S) con respecto a la inversión interna (I) (S-I), b) estimaciones (o hipótesis) sobre el nivel de equilibrio interno de mediano plazo, ya sea de CAP o S-I, y c) estimaciones (o hipótesis) sobre la relación de forma reducida entre el CUR, el tipo de cambio real (R) y otras variables. 
El MEM puede comprenderse a partir de algunas relaciones contables. En inicio, a partir de la identidad contable del ingreso nacional (Ec. 1) puede deducirse que la diferencia entre el ahorro y la inversión debe ser igual al saldo en la balanza comercial (Ec. 3).

$$
\begin{aligned}
& Y=C+I+G+X-M \\
& (Y-C-G)-I=X-M \\
& S-I=X-M
\end{aligned}
$$

Dónde: $Y=$ Renta, $C=$ Consumo, $I=$ Inversión interna, $G=$ Gasto de gobierno, $X=$ Exportaciones y $M=$ Importaciones. Por otro lado, este modelo se basa en la identidad de la balanza de pagos que relaciona el saldo de la cuenta corriente (CUR) con el flujo neto de capital privado y oficial (CAP):

$$
C U R-C A P=0
$$

Por último, el CAP puede ser considerado como el exceso de ahorro interno $(S)$ con respecto a la inversión interna $(I)$, es decir $S$-I.

Isard (2007) sugiere que para aplicar el MEM resulta útil definir el concepto de saldo en cuenta corriente subyacente (UCUR), que en este caso se define como el valor de CUR que se observaría al tipo de cambio real vigente en el mercado si todos los países estuviesen operando a pleno empleo o a su producto potencial (equilibrio interno), y si se hubiesen asimilado completamente las variaciones anteriores del tipo de cambio?.

Tal como se detalla en FMI (2008), el MEM puede ser comprendido a partir de una revisión conceptual. En el Gráfico 3, la curva con pendiente negativa muestra una relación entre la cuenta corriente subyacente y el tipo de cambio real, en la que un incremento del tipo de cambio real, que se mide a lo largo del eje vertical, corresponde a una apreciación de la moneda nacional. La apreciación real generalmente conduce a un descenso del valor de las exportaciones y a un aumento del valor de las importaciones, lo cual implica un descenso en

9 Éste es el concepto apropiado de saldo en cuenta corriente a mediano plazo relacionado con el tipo de cambio real vigente, ya que es un concepto que se ajusta en función del ciclo económico y reconoce que los efectos de las variaciones del tipo de cambio sobre los volúmenes y valores de las importaciones y exportaciones generalmente demoran algún tiempo en materializarse por completo (rezagos). 
la cuenta corriente (ello explica la pendiente negativa). La línea vertical representa el nivel de equilibrio de $S$-I, que en este caso se asume que es independiente del tipo de cambio real ${ }^{10}$.

El lugar geométrico donde se intersectan las curvas de UCUR y S-I determina el valor de equilibrio del tipo de cambio real $\left(R^{*}\right)$. El cálculo de $R^{*}$ parte de las estimaciones de i) saldo en cuenta corriente subyacente (UCUR1) asociado con el valor vigente del tipo de cambio real $\left(R_{1}\right)$,y ii) brecha entre el nivel de equilibrio de S-I y UCUR. Posteriormente, la pendiente de la línea UCUR se utiliza para estimar cuánto tendría que cambiar $R$ para cerrar la brecha, ceteris paribus.

\section{Gráfico 3: Marco conceptual de los fundamentos del TCR a mediano plazo} Tipo de cambio real (R)

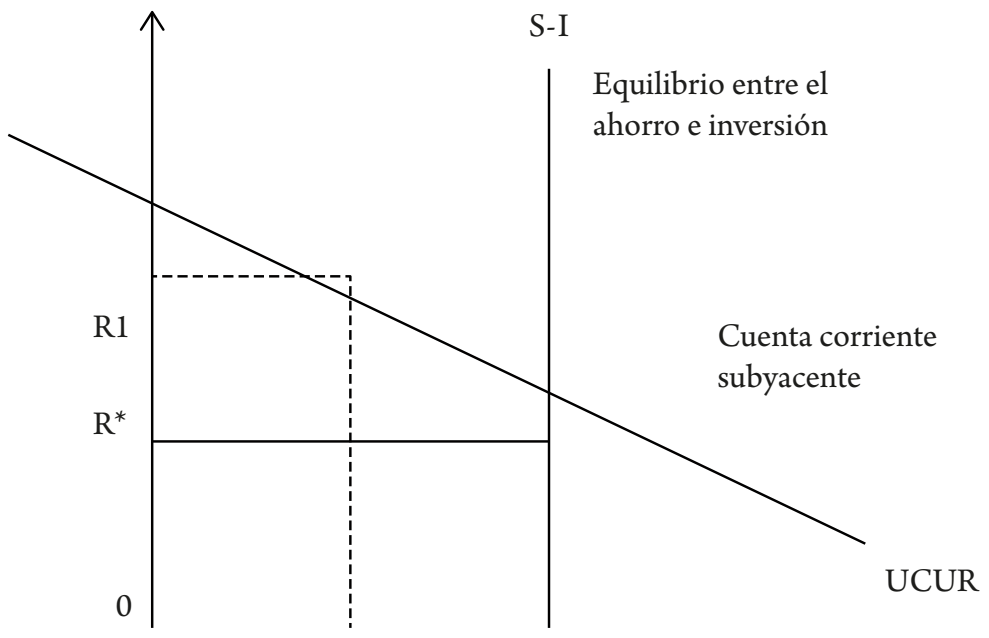

Déficit

Superávit

\section{UCUR1}

Nota: En el análisis teórico, el tipo de cambio real (R), siguiendo la notación de los textos consultados, corresponde a los precios nacionales/precios internacionales.

10 En general, los saldos de la línea UCUR y de la línea S-I dependerán de algunas variables no consideradas en el gráfico. 
La estimación del saldo de S-I de equilibrio plantea un desafío mayor que la estimación del saldo UCUR ${ }^{11}$. Un enfoque se basa en estimaciones econométricas de la relación entre el saldo S-I, aproximada por el saldo en cuenta corriente, y una lista de las variables explicativas pertinentes; y la otra, en el cálculo del saldo S-I de equilibrio o la "norma de la cuenta corriente" a partir de la relación estimada, basándose en supuestos acerca de los valores de equilibrio de las variables explicativas (FMI, 2008).

Debelle y Faruqee (1996) fueron los pioneros de este enfoque, ya que utilizaron datos de panel de los países industriales, centrándose principalmente en la manera en que los saldos S-I a nivel de toda la economía se ven afectados por la etapa del crecimiento económico (con un impacto negativo, porque las economías en fase de crecimiento suelen requerir financiación exterior), factores demográficos (crecimiento de la población y el porcentaje de personas dependientes, con un impacto negativo, pues una menor población potencialmente activa genera mayor consumo y una menor producción), y saldos fiscales estructurales (con un impacto favorable en la cuenta corriente, pues un superávit público implica ceteris paribus un mayor ahorro nacional).

Estudios posteriores incluyeron también la renta per cápita relativa (con un impacto positivo, porque las economías en una etapa más avanzada de desarrollo suelen tener capacidad de financiación para aquellas economías en fase de crecimiento), la posición de inversión internacional (con un impacto positivo, por la mejor balanza de rentas asociada a una posición acreedora) y la balanza energética (con un impacto positivo obvio).

Conociendo cuál es el impacto de esas variables sobre la cuenta corriente, se halla la "norma de la cuenta corriente" aplicando dichos estimadores a los valores de las variables mencionadas anteriormente para un horizonte determinado. Finalmente, usando las elasticidades de la cuenta corriente respecto al tipo de cambio, se calcula el cambio en el valor de éste que sería necesario para llevar a la cuenta corriente desde su valor actual a su valor "norma o de equilibrio".

Siguiendo la metodología descrita, a continuación se estima el des-alineamiento del tipo de cambio real de Bolivia para 2011. En inicio se explica mediante una regresión de datos de papel la cuenta corriente en los países de bajo ingreso. Esta regresión es obtenida de

11 En los ejercicios de evaluación del FMI se toman las estimaciones de UCUR a partir de las proyecciones a mediano plazo contenidas en Perspectivas de la Economía Mundial (WEO, por sus siglas en inglés) bajo los supuestos de que las brechas del producto se cierran y los tipos de cambio reales permanecen invariables. 
estimaciones realizadas por el FMI (2011) con datos de 1983-2007 para los países de ingresos bajos, según la clasificación de este organismo internacional (Cuadro 3).

\section{Cuadro 3}

Estimación de la cuenta corriente norma a partir de un modelo de panel para países de ingresos bajos

\begin{tabular}{lc}
\hline \hline VARIABLES & \\
\hline \hline & \\
Saldo fiscal/PIB & $0.2659^{* *}$ \\
& $(0.0179)$ \\
Tasa de dependencia & $-0.033^{6}$ \\
& $(0.8814)$ \\
Crecimiento de la Población & $-1.7194^{* *}$ \\
& $(0.0100)$ \\
Activos externo netos rezagados & -0.0129 \\
Saldo comercial petrolero/PIB & $(0.2357)$ \\
& $0.2188^{* *}$ \\
PIB percápita & $(0.0199)$ \\
& 0.0831 \\
Crecimiento PIB percápita & $(0.6901)$ \\
& 0.1719 \\
Flujo de asistecia/PIB & $(0.1647)$ \\
& $-0.1464^{* *}$ \\
Constante & $(0.0153)$ \\
& $-0.1842^{* * *}$ \\
Bolivia FE & 0.0000 \\
& $0.1562^{* * *}$ \\
Observaciones & 0 \\
R-cuadrado & \\
\hline \hline Robust p-values en parentesis & 249 \\
${ }^{* * *} \mathrm{p}<0.01,{ }^{* *} \mathrm{p}<0.05,{ }^{*} \mathrm{p}<0.1$ & 0.74 \\
& \\
& \\
& \\
&
\end{tabular}

Posteriormente, para proyectar la cuenta corriente, es decir, hallar la "norma de la cuenta corriente”, se evalúa este panel respecto a los parámetros fundamentales de la economía para 2011. Este valor nos permite encontrar la diferencia entre la norma de la cuenta corriente y la cuenta corriente (esta última proyección también se denomina "cuenta corriente subyacente" y se la puede observar en el Cuadro 4). 


\begin{tabular}{l} 
Cuadro 4 \\
Estimación de la brecha de la cuenta corriente respecto a la \\
\cline { 2 - 2 } \begin{tabular}{lc} 
Parámetro & Valar \\
\hline \hline Cuenta corriente que estabiliza los AEN $\left(\alpha c^{*}\right)$ & $-0.7 \%$ \\
Cuenta corriente subyacente ( $c$ en 2011) & $2.2 \%$ \\
Brecha de cuenta corriente & $-2.9 \%$ \\
\hline
\end{tabular}
\end{tabular}

Fuente: Estimaciones propias

Para calcular la variación del tipo de cambio que cerrará la brecha de la cuenta corriente se emplea la elasticidad de la cuenta corriente ( $c c)$ al TCR, que se calcula a partir de la siguiente ecuación:

$\eta_{c c}=\eta_{x} \times(x / y)-\eta_{m} \times(m / y)$

\section{Cuadro 5}

Estimación de la elasticidad de la cuenta corriente al tipo de cambio real

\begin{tabular}{lc}
\hline Parámetro & Valar \\
\hline \hline Elasticidad de las Exportaciones al TCR $\left(\eta_{x}\right)$ & 1.61 \\
Elasticidad de las Importaciones al TCR $\left(\eta_{m}\right)$ & 1.65 \\
Exportaciones / PIB $(x / y)$ & $0.3^{2}$ \\
Impartaciones / PIB $(m / y)$ & 0.27 \\
\hline \hline
\end{tabular}

Fuente: Cerezo y Salazar (2011)

Nota: Para la estimación de las elasticidades se consideró una muestra anual desde 1991 a 2010. Merece especial atención la elasticidad positiva de las importaciones al tipo de cambio real, hecho que podría deberse a la dinámica propia de los sectores que consideran a otras variables como relevantes, aparte del TCR.

Considerando la elasticidad de la cuenta corriente al tipo de cambio real no linealizado (0.06), se obtiene la variación necesaria en el tipo de cambio que hace que la cuenta corriente alcance a aquélla que estabilice los AEN: 
$\eta_{C A}=\frac{\Delta \% C C}{\Delta \% T C R}$

$\Delta \% T C R=\frac{\Delta \% C C}{\eta_{C A}}=\frac{-0.029}{0.06}=-0.48$

Los resultados del Método de Equilibrio Macroeconómico (MEM) sugieren que es necesaria una apreciación real de $0.48 \%$ para que la cuenta corriente registrada en 2011 sea compatible con la norma de la cuenta corriente. Este resultado depende de la cuenta corriente subyacente del $2.2 \%$ del PIB y una norma de la cuenta corriente estimada en un $-0.7 \%$ del PIB. El nivel de la norma de la cuenta corriente refleja principalmente la reducción en el superávit fiscal y el crecimiento del PIB per cápita, con el consecuente incremento en las importaciones.

\subsection{Método de Sostenibilidad Externa (MSE)}

El Método de Sostenibilidad Externa estima el saldo de la cuenta corriente que estabiliza la posición de inversión internacional (como porcentaje del PIB) en un determinado nivel (CCAEN). Este nivel puede establecerse de manera arbitraria (por ejemplo, podría ser el valor actual) o puede también ligarse a una determinada estimación empírica sobre el valor de la posición de inversión internacional en "equilibrio" en función de una serie de fundamentos (parecido a lo que hace el MEM para la cuenta corriente de "equilibrio").

Este MSE tiene la ventaja de que no requiere a priori una estimación cuantitativa, aunque sí una serie de proyecciones sobre algunas variables, como el crecimiento, la inflación o el rendimiento de activos y pasivos, necesarios para predecir las dinámicas futuras de la posición de inversión internacional ${ }^{12}$.

Con el saldo de la cuenta corriente que estabiliza la posición de inversión internacional estimado, se calcula el ajuste necesario en el tipo de cambio que llevaría a la cuenta corriente desde su valor actual al valor que estabiliza la posición de inversión internacional, usando nuevamente la elasticidad de la cuenta corriente al tipo de cambio real, como se hizo en el MEM.

12 También se precisan ciertos supuestos, como la no variación en el precio de activos y pasivos exteriores o la ausencia de transferencias de capital o errores y omisiones, de forma que las variaciones en la posición de inversión internacional sólo se explican por la cuenta corriente o el tipo de cambio. 
Para poder realizar las estimaciones por el MSE se requieren tres cálculos complementarios:

a) Determinar un nivel de cuenta corriente que estabiliza los activos externos netos (CCAEN).

b) Calcular la cuenta corriente para un año determinado, valorando las mismas cantidades físicas observadas, a los precios de tendencia para las principales exportaciones e importaciones (este cálculo corresponde a la cuenta corriente subyacente (UCUR) ${ }^{13}$ ).

c) Determinar la depreciación o apreciación real requerida para que la CCE sea igual a la UCUR. El TCR de equilibrio corresponde al TCR observado durante el período analizado, corregido por la depreciación o apreciación requerida ${ }^{14}$.

Las implicaciones del MSE para las diferentes economías son sencillas. Economías deudoras que tienen una alta tasa de crecimiento pueden experimentar un déficit en corriente más prolongado, sin que implique un aumento en la razón pasivos externos/PIB. Además, altas tasas de rendimiento sobre los activos y pasivos externos implica que los países deudores necesitan grandes ajustes en la balanza comercial para estabilizar la posición externa. Por último, las economías que perciben menores tasas de retorno sobre sus activos de lo que pagan por sus deudas (por ejemplo, debido a las primas de riesgo de su deuda externa), requieren mayores superávit comerciales para estabilizar la posición de sus activos externos netos.

La base teórica parte de la ecuación de acumulación de activos externos netos en una economía:

$$
C C_{t}=B_{t}-B_{t-1}=T B_{t}+G R_{t}+r B_{t-1}
$$

Donde $C C_{t}, B_{t}, T B_{t}, G R_{t}$ y $r$ son la cuenta corriente, los activos externos netos, el saldo comercial, las donaciones externas o remesas y la tasa de interés, respectivamente.

Si se asume una tasa de crecimiento del PIB a largo plazo como una constante " $g$ ", se tiene:

$$
Y_{t}=(1+g) Y_{t-1}
$$

\footnotetext{
13 La cuenta corriente subyacente se puede obtener a partir de las estimaciones de organismos internacionales, como el FMl, que siguen esta metodología.

14 Este método simple hace que sea una referencia natural para comparar los otros métodos sofisticados que usan
} modelos econométricos. 
Operando con las ecuaciones 8 y 9 se deriva la ecuación de acumulación de activos externos netos como coeficiente del PIB, la cual denotamos para todas las variables con letras minúsculas:

$b_{t}-b_{t-1}=t b_{t}+g r_{t}+\frac{(r-g)}{1+g} b_{t-1}$,

En línea con Cerezo y Salazar (2011), a continuación se presenta un ejercicio para Bolivia que compara el saldo UCUR asociado con el tipo de cambio real vigente con una estimación del saldo en cuenta corriente requerido para estabilizar el nivel actual (o proyectado) y la composición de los pasivos externos netos. En inicio se deriva la ecuación del saldo comercial $\left(t b^{*}\right)$ que estabiliza el coeficiente de activos externos netos/PIB (es decir: $b_{t}=b_{t-1}=b$ ). Operando en la identidad 10 tenemos:

$t b *=-\frac{r-g}{1+g} b-$ grant

En el siguiente cuadro se estima $t b^{*}$ a partir de sus determinantes para 2011:

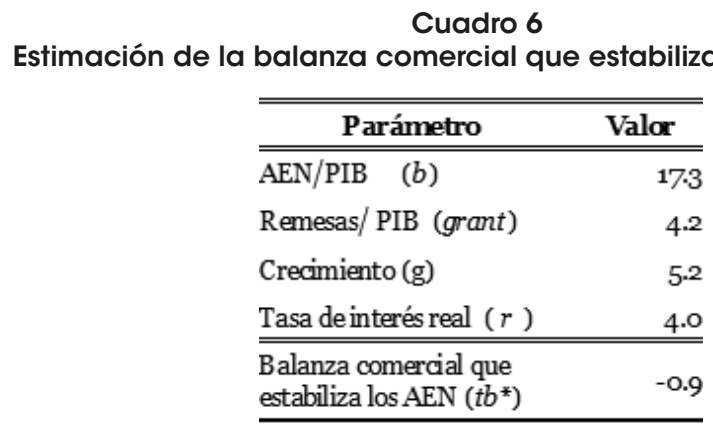

Fuente: Elaboración propia

Posteriormente, para calcular el saldo en cuenta corriente que estabilizará los activos externos netos $\left(c c^{*}\right)$ en el nivel registrado en 2011 , se emplea la identidad 12: 
$c c *=t b *+$ grant $+\frac{r b}{1+g}$

Con la información contenida en el Cuadro 7 y con la estimación de la $t b^{*}$ se obtiene que la cuenta corriente que estabilizará los activos externos netos es $\mathrm{cc}^{*}=-0.9$. Para completar el análisis se obtiene la Cuenta Corriente Subyacente (UCUR) y se calcula la brecha de esta respecto a la que estabiliza el AEN. La información relevante se encuentra en la siguiente tabla:

\section{Cuadro 7}

Estimación de la brecha de la cuenta corriente

\begin{tabular}{lr}
\hline Parámetro & Valor \\
\hline Cuenta corrierte que estabiliza los AEN (cc*) & -0.9 \\
Cuenta corrierte subyacente (cc en 2011) & 4.2 \\
\hline Brecha en la cuenta corriente & -5.1 \\
\hline
\end{tabular}

Nota: Para el cálculo de la cuenta corriente subyacente se considera el valor observado para 2011

Finalmente, para calcular la variación del tipo de cambio real necesaria para cerrar la brecha entre la $c c^{*}$ y la cc en 2011 se emplea la elasticidad de la cuenta corriente (cc) al TCR que ya se estimó en el MEM (Cuadro 5). Considerando la identidad de la elasticidad de la cuenta corriente al tipo de cambio real no linealizada, se obtiene la variación necesaria en el tipo de cambio que hace que la cuenta corriente alcance a aquélla que estabilice los AEN es de $0.85 \%$. Es decir, el Método de Sostenibilidad Externa (MES) sugiere una subvaluación cambiaria de $0.85 \%$.

\section{Síntesis de los resultados}

La presente sección resume algunos hallazgos que se obtuvieron de la aplicación de los distintos métodos para estimar el desalineamiento cambiario boliviano para 2011. Inicialmente hay que destacar que los cuatro métodos empleados arrojan resultados similares, cuyo promedio data de una subvaluación reducida de $0.69 \%$. 
Cuadro 8

Desviación del tipo de cambio real respecto a su nivel de equilibrio en 2011 (en porcentajes)

\begin{tabular}{llc}
\hline \hline A & Filtro estadístico - Hodrick y Prescott & -0.78 \\
B & Modelo de Compartamiento del Tipo de Cambio Real & -0.65 \\
C & Método de Equilibrio Macroeconómico & -0.48 \\
D & Método de Sostenibilidad Externa & -0.85 \\
\hline \hline & Promedio (A, B, C y D) & -0.69 \\
\hline \hline
\end{tabular}

\author{
Fuente: Elaboración propia \\ Nota: (+) subvaluación, (-) sobrevaluación
}

Los resultados bajo el método estadístico (HP) dan cuenta de una subvaluación cambiaria de $0.78 \%$, y los del Método de Comportamiento del TCR, una de $0.65 \%$. Por otra parte, los métodos de Sostenibilidad Externa y de Equilibrio Macroeconómico reportan una subvaluación del tipo de cambio de 0.85 y $0.48 \%$, respectivamente.

\title{
4. Conclusiones
}

El tipo de cambio real es un precio relativo muy importante en una economía y el entendimiento de su comportamiento gana especial atención tanto en las economías desarrolladas como en las emergentes, debido a que desalineaciones del tipo de cambio real, diferencias respecto a su valor de equilibrio, afectan de manera significativa el proceso de asignación de recursos al modificar la rentabilidad relativa entre los sectores transables y no transables.

En ese sentido, el presente documento, a partir de la aplicación de diferentes métodos, estimó el tipo de cambio real de equilibrio para posteriormente identificar periodos de des-alineamiento y/o cuantificar la misma para el último periodo observado (2011). El primer enfoque se basó en un filtro estadístico (Hodrick y Prescott) comúnmente utilizado para extraer el componente de largo plazo de las series y el segundo enfoque se basó en un Modelo de Comportamiento del TCR. Ambos métodos identificaron tres periodos de desalineamiento: subvaluación real en el periodo 1994-1995 y sobrevaluación en 2002 y 2008-2009. Estos desequilibrios han estado asociados principalmente a desajustes macroeconómicos internacionales que afectaron a algunos países socios comerciales e 
indirectamente a la economía boliviana. La reciente crisis económica financiera internacional, que se inició aproximadamente en septiembre de 2008, fue el último hecho destacable que afectó al comportamiento de TCR.

Adicionalmente se estimó el desalineamiento cambiario para 2011 a partir de los métodos de Equilibrio Macroeconómico (MEM) y de Sostenibilidad Externa (MSE), los cuales fueron desarrollados por el FMI y son aplicados en la evaluación de la paridad cambiaria en sus diferentes informes. Específicamente, esta metodología sobre el tipo de cambio diseñada por el Grupo Consultivo (CGER, Consultative Group on Exchange Rate Issues) del FMI se basa en el MEM, MSE y Modelo de Comportamiento del Tipo de Cambio Real.

Los resultados de los cuatro métodos de estimación del tipo de cambio real de equilibrio mencionados sugieren una subvaluación cambiaria promedio de $0.69 \%$ para 2011. Todos los enfoques mostraron una subvaluación cambiaria baja, inferior al $1 \%$ a pesar de que cada metodología implica simplificaciones conceptuales e información diversa.

Finalmente, es importante destacar el seguimiento que sele debe hacer a este precio relativo, más aun considerando que ante un eventual desalineamiento persistente o fuerte es muy poco lo que se puede hacer a partir de un ajuste en el tipo de cambio nominal, considerando que la economía boliviana tiene un régimen cambiario administrado (crawling peg).

Artículo recibido: 14 de agosto de 2012 Manejado por: $A B C E$ Aceptado: 20 de septiembre de 2012 


\section{Referencias}

1. Ades, Alberto; Masih, Rumi y Tenengauzer, Daniel. (1999). "GSDEEMER". Third Edition.

2. Aguilar, M.A. (2003). Estimación del tipo de cambio real de equilibrio para Bolivia. Revista de Análisis del BCB, 6 (1)

3. Arellano, S. y Larraín, F. (1996). Tipo de cambio real y gasto público: un modelo econométrico para Chile. Cuadernos de Economía 33(9), 47-75.

4. Aukrust, D. (1977). Inflation in the open economy: A Norwegian model. Washington D.C.: Brookings:

5. Balassa, B. (1964). The purchasing power parity doctrine: A reappraisal. Journal of Political Economy 72, 584-596.

6. Baxter, Marianne y Crucini, Mario J. (1992). Business cycles and the asset structure of foreign trade. Institute for Empirical Macroeconomics 59, Federal Reserve Bank of Minneapolis. Discussion Paper.

7. Bello, O.; Heresi, R.y Pineda, R. (2010) El tipo de cambio real de equilibrio: un estudio para 17 países de América Latina. Macroeconomía del desarrollo, CEPAL, Serie 82.

8. Caputo, R. y Núñez, M. (2008). Tipo de cambio real de equilibrio en Chile: enfoques alternativos. Economía Chilena 11 (22), agosto.

9. Cerezo S.; Humerez, J. y Cossio. J. (2010). El desempeño del régimen cambiario boliviano en el periodo post-estabilización. Documento de trabajo, BCB.

10. Cerezo S. y Salazar, D. (2011). Tipo de cambio real de equilibrio en Bolivia: cinco enfoques alternativos. Documento de trabajo presentado en el 4EEB-BCB en la ciudad de Sucre.

11. Cerda, R.; Donoso, A. y Lema, A. (2005). Análisis del tipo de cambio real: Chile 19861999. Cuadernos de Economía, 42 (126), 329-56, noviembre.

12. Cerruti, E. y Mansilla, M. (2008). Bolivia and Its Booming Gas Sector: Should We Worry About a New Case of Dutch Disease? IMF Working Paper, No. 08/154, junio.

13. Choudhri, Ehsan y Khan, Mohsin (2004). Real Exchange Rates in Developing Countries: Are Balassa-Samuelson Effects Present? IMF Working Paper 04/188. International Monetary Fund. 
14. Debelle, G. y Faruqee, H. (1996). What Determines the Current Account? A CrossSectional and Panel Approach. IMF Working Paper 96/58.

15. Ferreyra, J. y Herrada, R. (2003). Tipo de cambio real y sus fundamentos: estimación de la desalineación. Revista Estudios Económicos No 10. Banco Central de Reserva del Perú.

16. Fondo Monetario Internacional, FMI (2008). Por una economía mundial. Informe anual.

17. Fondo Monetario Internacional, FMI (2011): Bolivia 2011 Article IV Consultation Cover, pag 21.

18. Frenkel, J. y Razin, A. (1986). Real exchange rates, interest rates and fiscal policies. Economic Studies Quarterly, 37, 99-113.

19. Frenkel, J. y Razin, A. (1987). Fiscal Policies and the World Economy, Cambridge, Mass.: MIT press.

20. Hodrick, Robert J. y Prescott, E.C. (1980). Postwar U.S. Business Cycles: an Empirical Investigation. mss. Pittsburgh: Carnegie-Mellon University; Discussion Papers 451, Northwestern University

21. Hoffmann, Mathias y MacDonald, Ronald (2003). A Re-examination of the Link Between Real Exchange Rate and Real Interest Rate. CESIFO, Working Paper No. 894.

22. Isard, P. (2007). Equilibrium Exchange Rates: Assessment Methodologies. IMF Working Paper N07/296.

23. Lee, J.; Milesi-Ferretti, G. M.; Ostry, J.; Prati, A. y Ricci, L. A. (2006). Methodology for CGER Exchange Rate Assessments. Fondo Monetario Internacional.

24. Lee, J.; Milesi-Ferretti, G. M.; Ostry, J.; Prati, A. y Ricci, L. A. (2008). Exchange Rate Assessments: CGERMethodologies. IMF Occasional Paper, No 261, Fondo Monetario Internacional.

25. Lee, J.; Milesi-Ferretti, G. A. y Ricci, L. A. (2008). Real Exchanges and Fundamentals: A Cross-Country Perspective. IMF Working Paper, WP/08/13, Fondo Monetario Internacional.

26. Lora, O. y Orellana, W. (2000). Tipo de cambio real de equilibrio: un análisis del caso boliviano en los últimos años. Revista de Análisis del BCB, 3 (1). 
27. MacDonald, Ronald y Ricci, Luca (2003). Estimation of the Equilibrium Exchange Rate for South Africa. IMF Working Paper 03/44. International Monetary Fund.

28. Mendieta, P. (2007). El equilibrio de la competitividad cambiaria boliviana: un enfoque empírico. Documento presentado en la Reunión del Proyecto Conjunto sobre Variables no Observables, llevado a cabo en Buenos Aires el 15 de junio de 2007

29. Miyajima, Ken (2005). Real Exchange Rates in Growing Economies: How Strong Is the Role of the Nontradables Sector? IMF Working Paper 05/233. International Monetary Fund.

30. Obstfeld, M. (1981). Macroeconomic Policy, Exchange-Rate Dynamics, and Optimal Asset Accumulation. Journal of Political Economy, University of Chicago Press, 89(6), 1142-61, December.

31. Svensson, L. y Razin, A. (1983). The Terms of Trade and the Current Account: The Harberger-Laursen-Metzler Effect. Journal of Political Economy, 91, 97-125.

32. Williamson, John (1983). The Exchange Rate System. Policy Analyses in International Economics 5. Washington: Institute for International Economics. 


\section{Anexos}

\section{A1. Análisis de raíz unitaria}

Los resultados del test de $\mathrm{ADF}$ muestran que se acepta la hipótesis de que las variables son integradas de orden uno, incluyendo una constante y/o tendencia según sea el caso de cada variable.

Todas las series fueron desestacionalizadas por X12-Arima; el periodo de análisis abarca de 1990Q1 a 2011 Q4.

Cuadro A1.1

Análisis de raíz unitaria de las variables (Dickey-Fuller aumentado)

\begin{tabular}{lcccc}
\hline & \multicolumn{2}{c}{ Niveles } & \multicolumn{2}{c}{ Diferencias } \\
\cline { 2 - 5 } Descripción & Estadístico t 1/ & Rezagos a/ & Estadístico t 1/ & Rezagos a/ \\
\hline Ln(Balance Fiscal/PIB) & -3.33 & 1 & -13.24 & 0 \\
Ln(Balanza Comercial/PIB) & -3.96 & 1 & -12.41 & 1 \\
Ln(Términos de Intercambio) & -2.98 & 1 & -9.40 & 1 \\
Ln(Precio Gas) & -4.13 & 1 & -5.58 & 1 \\
Ln(RN) & -3.45 & 1 & -12.74 & 1 \\
\hline \hline
\end{tabular}

Notas:

1 Una constante y tendencia es incluida en cada estimación. Los valores críticos son: -4.07 para $1 \%,-3.47$ para $5 \%$ y-3.16 para $10 \%$.

2 Basado en el criterio de Schwarz con un máximo de 10 rezagos. 
A2. Análisis de cointegración

\section{Cuadro A2.1 \\ Tests de Cointegración Johansen -Modelo TCRE}

\begin{tabular}{|c|c|c|c|c|c|}
\hline \multicolumn{6}{|c|}{ (1) } \\
\hline$\lambda$ trace & prob. & & $\lambda$ max & prob. & \\
\hline 25.43 & 0.68 & & 12.74 & 0.80 & \\
\hline 13.89 & 0.63 & & 8.46 & 0.77 & \\
\hline 6.63 & 0.50 & & 6.52 & 0.43 & \\
\hline 1.32 & 0.76 & & 1.32 & 0.76 & \\
\hline \multicolumn{6}{|c|}{ (2) } \\
\hline $\begin{array}{c}\lambda \text { trace } \\
13.16\end{array}$ & $\begin{array}{r}\text { prob. } \\
0.12\end{array}$ & \multirow[t]{4}{*}{$\$ *$} & $\begin{array}{l}\lambda \max \\
16.27\end{array}$ & $\begin{array}{l}\text { prob. } \\
0.11\end{array}$ & \multirow[t]{4}{*}{$* *$} \\
\hline 11.09 & 0.34 & & 8.79 & 0.54 & \\
\hline 3.50 & 0.40 & & 3.25 & 0.70 & \\
\hline 1.45 & 0.58 & & 1.61 & 0.58 & \\
\hline \multicolumn{6}{|c|}{ (3) } \\
\hline$\lambda$ trace & prob. & \multirow{5}{*}{$* *$} & $\lambda_{\lambda \max }$ & prob. & \multirow{5}{*}{ * } \\
\hline 58.08 & 0.09 & & 3261 & 0.04 & \\
\hline 26.67 & 0.60 & & 13.18 & 0.77 & \\
\hline 14.69 & 0.56 & & 9.16 & 0.70 & \\
\hline 6.73 & 0.47 & & 6.89 & 0.38 & \\
\hline \multicolumn{6}{|c|}{ (4) } \\
\hline$\lambda$ trace & prob. & \multirow{6}{*}{$* *$} & $\lambda_{\text {max }}$ & prob. & \\
\hline 61.10 & 0.00 & & 38.81 & 0.01 & $*$ \\
\hline 41.21 & 0.02 & & 28.68 & 0.02 & \\
\hline $25 \cdot 32$ & 0.29 & & $12.3^{8}$ & 0.36 & \\
\hline 13.36 & 0.41 & & 7.03 & 0.36 & \\
\hline 5.17 & $0.6_{3}$ & & 1.50 & 0.63 & \\
\hline
\end{tabular}

Nota: Las estimaciones incluyen 4 rezagos y una constante. ${ }^{*}=10 \%,{ }^{* *}=5 \%{ }^{* * *}=1 \%$. 